\title{
Identification of novel PKD1 and PKD2 mutations in Korean patients with autosomal dominant polycystic kidney disease
}

Rihwa Choi ${ }^{1 \dagger}$, Hayne Cho Park ${ }^{2 \dagger}$, Kyunghoon Lee ${ }^{1}$, Myoung-Gun Lee ${ }^{1}$, Jong-Won Kim ${ }^{1}$ Chang-Seok Ki ${ }^{*}$, Young-Hwan Hwang ${ }^{3^{*}}$ and Curie Ahn ${ }^{2}$

\begin{abstract}
Background: Autosomal dominant polycystic kidney disease (ADPKD) is the most common inherited kidney disorder. It is caused by mutations in the PKD1 and PKD2 genes, and manifests as progressive cyst growth and renal enlargement, resulting in renal failure. Although there have been a few studies on the frequency and spectrum of mutations in PKD1 and PKD2 in Korean patients with ADPKD, only exons 36-46, excluding the duplicated region, were analyzed, which makes it difficult to determine accurate mutation frequencies and mutation spectra.

Methods: We performed sequence analysis of 20 consecutive unrelated ADPKD patients using long-range polymerase chain reaction (PCR) to avoid pseudogene amplification, followed by exon-specific PCR and sequencing of the all exons of these two genes. Multiplex ligation-dependent probe amplification was performed in patients in whom pathogenic mutations in PKD1 or PKD2 were not identified by LR-PCR and direct sequencing to detect large genomic rearrangements.

Results: All patients met the diagnostic criteria of ADPKD, and pathogenic mutations were found in 18 patients (90.0\%), comprising 15 mutations in PKD1 and three in PKD2. Among 10 novel mutations, eight mutations were found in the PKD1 gene while two mutations were found in the PKD2 gene. Eight of 14 PKD1 mutations (57.1\%) were located in the duplicated region.

Conclusions: This study expands the spectra of mutations in the PKD1 and PKD2 genes and shows that the mutation frequencies of these genes in Korean ADPKD patients are similar to those reported in other ethnicities. Sequence analysis, including analysis of the duplicated region, is essential for molecular diagnosis of ADPKD.
\end{abstract}

Keywords: Autosomal dominant polycystic kidney disease, Korean, Mutation, PKD1, PKD2

\section{Background}

Autosomal dominant polycystic kidney disease (ADPKD) is the most common inherited kidney disorder, affecting approximately 1 in 400 to 1,000 individuals in Western countries. It is characterized by progressive cyst growth and renal enlargement, resulting in end stage renal disease (ESRD) [1]. ADPKD is caused by mutations in the

\footnotetext{
* Correspondence: changski@skku.edu; ondahl@eulji.ac.kr

${ }^{\dagger}$ Equal contributors

'Department of Laboratory Medicine and Genetics, Samsung Medical Center, Sungkyunkwan University School of Medicine, (135-710) 81 Irwon-Ro Gangnam-gu, Seoul, South Korea

${ }^{3}$ Department of Internal Medicine, Eulji General Hospital, (139-872), 1306

Dunsan 2(i)-dong, Seo-gu, Daejeon, Seoul, South Korea

Full list of author information is available at the end of the article
}

PKD1 (16p13.3) and PKD2 (4q21) genes, which produce the proteins polycystin-1 (PC-1) and PC-2, respectively. These proteins have critical roles in maintaining normal renal tubular structure during kidney development. These proteins have also been found in primary cilia, where they function as mechanosensors between cells, or at cell-matrix attachments, where they mediate cell adhesion [2]. In clinically defined populations, mutations in PKD1 and PKD2 account for $\sim 85 \%$ and $\sim 15 \%$ of ADPKD cases, respectively [1,3]. Patients with PKD1 mutations exhibit a more progressive renal phenotype than those with PKD2 mutations (average age at onset of ESRD, 53.4 years vs. 72.7 years, respectively) [4]. 
ADPKD is typically diagnosed by renal imaging with age-related cyst number criteria; however, imaging cannot exclude disease in at-risk individuals under 40 years of age $[5,6]$. Mutation-based diagnostics are increasingly being used in individuals who are at risk of ADPKD, and have proven especially helpful in cases where imaging studies are equivocal and a definitive diagnosis is required, such as when evaluating potential living related kidney donors [7]. In addition, PKD1 and PKD2 mutation analyses can potentially provide prognostic as well as diagnostic information, such as pre-implantation genetic diagnostics for early-onset ADPKD with the advent of effective therapies for ADPKD $[7,8]$.

Mutation analysis of ADPKD has been hampered by the large sizes and multi-exon structures of PKD1 and $P K D 2$, genomic duplication of PKD1, marked allelic heterogeneity, and common missense variants with hypomorphic alleles [7]. Two-thirds of the PKD1 gene (exons $1-33$ ) is duplicated six times on chromosome 16 (pseudogenes PKD1P1-P6), which share $97.7 \%$ sequence identity with $P K D 1$ but carry large deletions compared with PKD1 [9]. To analyze exons 1-33 of PKD1, long-range PCR (LR-PCR) needs to be performed before sequencing procedures [10]. Although several indirect methods have been developed, such as denaturing gradient gel electrophoresis (DGGE), single-strand conformation polymorphism (SSCP) analysis, protein-truncation tests, denaturing high performance liquid chromatography (DHPLC), transgenomics SURVEYOR nuclease and WAVE nucleic acid high sensitivity fragment analysis, and high resolution melt analysis, direct sequencing is undoubtedly the gold standard [3]. Quantitative PCR, multiplex ligation-dependent probe amplification (MLPA), and array-comparative genomic hybridization (array-CGH) can also aid in the detection of large genomic rearrangements [3,11].

Studies using different molecular diagnostic methods have reported different mutation detection rates among different populations [1,3,12-17]. Although there are a few studies on the frequency and spectrum of mutations in the PKD1 and PKD2 genes in Korean patients with ADPKD, only exons 36-46 of PKD1 were analyzed, making it difficult to determine accurate mutation frequencies and spectra [18-20]. In this study, we performed LR-PCR followed by nested PCR and direct sequencing as well as MLPA of PKD1 and PKD2 in 20 unrelated Korean ADPKD patients, covering all exons and flanking intronic regions for the first time.

\section{Methods}

We analyzed 20 unrelated patients with ADPKD from April 2010 to April 2014. The diagnosis of ADPKD was made according to previously described renal ultrasound criteria, including a family history of ADPKD [5,6]. Blood samples were obtained after receiving informed consent. This study was approved by the Institutional Review Board of Samsung Medical Center.

Genomic DNA was extracted from peripheral blood samples using a commercially available extraction kit (Promega, Madison, WI, USA). For the duplicated region of PKD1, LR-PCR was performed using PKD1-specific primers as described previously [21]. Direct sequencing of exonic and flanking introgenic regions of all PKD1 and PKD2 exons was performed using previously-described PCR primers and conditions [21]. PCR products were purified and sequenced on an ABI3730xl Genetic Analyzer (Applied Biosystems, Foster City, CA, USA).

MLPA was performed in patients in whom pathogenic mutations were not identified in PKD1 or PKD2 by LRPCR and direct sequencing to detect large genomic rearrangements. Deletion/duplication analysis of PKD1/ PKD2 exons was performed using the MLPA kit (SALSA MLPA KIT P351-B2/P352-C1 PKD1-PKD2; MRC-Holland, Amsterdam, The Netherlands) according to the manufacturer's instructions. This kit contains PKD1 probes for 37 of the 46 exons and covers all 15 PKD2 exons.

The 1000 Genomes Project data (http://browser.1000 genomes.org), the National Heart, Lung, and Blood Institute (NHLBI) Exome Sequencing Project (ESP) database (http://evs.gs.washington.edu/EVS), the NCBI database of Single Nucleotide Polymorphisms (dbSNPs), and the ADPKD Mutation Database (http://pkdb.mayo.edu, PKDB) were checked for previously reported sequence variants.

The pathogenicity of missense variants was evaluated by in silico analyses using Sorting Intolerant from Tolerant (SIFT) (http://sift.jcvi.org/), Polymorphism Phenotyping v.2 (PolyPhen-2) (http://genetics.bwh.harvard.edu/pph2/), and the Grantham matrix scoring system Align Grantham Variation and Grantham Deviation (Align-GVGD) (http:// agvgd.iarc.fr/) prediction programs, as described previously [1]. Human Splice Finder software (http://umd.be/ $\mathrm{HSF} /$ ) was used to predict splicing signals [22].

Variations identified in the current study were categorized into five classes: definite pathogenic mutation, likely pathogenic mutation, variant of unknown significance (VUS), likely benign variant, and benign [23]. The first class refers to nonsense mutations, frameshift deletions, insertions or indels, typical splicing mutations, or in-frame changes of $\geq 5$ amino acids detected by direct sequencing, as well as large genomic rearrangements detected by MLPA. In-frame changes of $<5$ amino acids, as well as missense and atypical splicing variants previously reported in patient(s) with sufficient evidence to classify them as pathogenic were also classified as definite pathogenic mutations. The second class refers to novel missense variants with the potential of pathogenicity as evaluated by in silico analyses with agreement among various methods, which included 'Not tolerated' on SIFT, 'Probably damaging' or 'Possibly damaging' on 
PolyPhen-2, and a score of C55 to C66 on AlignGVGD. Variations previously reported as 'likely pathogenic', but without sufficient evidence, were also classified as the second class. The third class refers to novel variants (synonymous or missense variants) predicted not to affect protein function by any of the in silico analyses, and intronic variations predicted not to alter splicing that were absent from the 1000 Genome Project data, ESP database of more than 13,000 control alleles, and an in-house collection of 150 exomes, or previously reported variations termed 'indeterminate' on PKDB. The fourth class refers to novel synonymous variations not reported previously that were found in control studies, suggesting that they are rare benign polymorphisms. The last class refers to benign SNPs. The recommendations of the Human Genome Variation Society for mutation nomenclature were followed. Nucleotide changes were numbered according to the coding sequences of PKD1 (NM_001009944.2) and PKD2 (NM_000297.3).

\section{Results}

During the 4-year study period, we performed mutational analysis using LR-PCR, followed by nested PCR and direct sequencing of PKD1 and PKD2 in 20 unrelated Korean patients diagnosed with ADPKD by ultrasound. Four of 20 PKD patients with an unknown or no family history of polycystic kidney disease had enlarged kidneys with more than 10 cysts in each kidney. There were 15 male and five female patients. The mean ( \pm S.D.) age was $42.6( \pm 8.4)$ years.

We identified 20 different variants in PKD1 and PKD2 among 20 ADPKD patients, including 17 pathogenic sequence variants, two VUS identified by sequence analysis, and one large deletion identified by MLPA analysis. Among these variants, 18 were classified as pathogenic mutations (7 frameshift, 6 nonsense, 3 missense, 1 splicing, and 1 large deletion). For six of seven patients who were not identified as having definite pathogenic mutations in sequence-based mutation analysis, MLPA analyses were performed. In one patient who was identified as having a likely pathogenic mutation of c.11541C > G (p.Ser3847Arg) in PKD1, MLPA analysis could not be performed because additional samples were not available. Each ADPKD patient had a unique mutation in $P K D 1$ or $P K D 2$. We found disease-causing mutations in patients with an overall mutation detection rate of $90.0 \%$ $(18 / 20)$; $94.4 \%(17 / 18)$ patients were identified by LRPCR and direct sequencing. The remaining one patient (5.6\%) was identified as having a PKD1 mutation with large genomic rearrangements (deletion of one copy of the whole PKD1 gene) by MLPA.

Among the 18 patients shown to have pathogenic mutations, $15(83.3 \%)$ patients had mutations in PKD1 (6 frameshift, 4 nonsense, 3 missense, 1 splicing, and 1 large deletion) and three (16.7\%) patients had two nonsense and one frameshift PKD2 mutations (Tables 1 and 2). Thirteen of the 18 (72.2\%) pathogenic mutations were nonsense or frameshift mutations: $66.7 \%(10 / 15)$ in PKD1 and $100 \%$ (3/3) in PKD2. All three missense variants found in PKD1 were classified as likely pathogenic mutations and were predicted to affect protein function by all three insilico analyses. Ten of 18 (55.6\%) mutations were not previously reported, including eight mutations in the PKD1 gene and two mutations in the PKD2 gene. Among 14 PKD1 mutations identified by LR-PCR and direct sequencing, eight (57.1\%) mutations were located in the duplicated region, outside the range of exons 36-46, and were not covered in previous reported studies in Korea.

Two novel VUSs that were identified in patients without known PKD mutations are summarized in Table 3. The novel intronic variation of c. $595+15 \mathrm{C}>\mathrm{T}$, which was found in $P K D 2$, was predicted to have no effect on normal splicing but was not identified in healthy controls from the 1000 Genome Project data, the ESP database of more than 13,000 control alleles, or an in-house collection of 150 exomes. Another novel synonymous variant of c.11604G >C (p.Thr3868=) was found in $P K D 1$ exon 42. The question of whether this variant is a rare SNP or a disease-associated pathogenic variant could not be easily addressed; although its effect on mRNA splicing was not examined in vitro, HSF predicted it would affect an enhancer motif.

\section{Discussion}

To the best of our knowledge, this is the first study to identify PKD1 and PKD2 mutations based on analysis of all coding exons and flanking regions in Korean ADPKD patients using LR-PCR followed by direct sequencing and MLPA.

The mutation detection rate $(90.0 \%)$ in our study $(83.3 \%$ in PKD1 and $16.7 \%$ in PKD2) was comparable to previous studies using LR-PCR followed by nested PCR and direct sequencing in clinically well-defined large cohorts in Western countries [1,3]. Although the proportion of PKD2 mutations has been reported to be $23 \%$ in Asian populations [16,17], PKD2 mutations accounted for $16.7 \%$ of the total mutations in our study subjects, comparable to data from Western countries (15.0\%-16.2\%) $[1,3]$. In contrast, a recent population-based study (Olmsted County population study) [24] and studies in an outpatient clinic setting $[25,26]$ reported a higher PKD2 mutation detection rate than earlier studies, which recruited large families with a history of ERSD. Future studies of a larger cohort of patients are needed to further understand the influence of ethnicity on genetic and/or phenotypic characteristics of ADPKD patients.

Among 14 pathogenic PKD1 mutations (excluding one large gene deletion), eight $(57.1 \%)$ were located outside 
Table 1 Definitive pathogenic mutations in PKD1 and PKD2 found in this study

\begin{tabular}{|c|c|c|c|c|c|c|c|}
\hline Gene & Exon/Intron & cDNA change & Amino acid change & Known/Novel & Type & Family history & Reference \\
\hline PKD1 & 12 & c.2865delC & p.Val956Trpfs*20 & Known & Frameshift & + & PKDB [3] \\
\hline PKD1 & 12 & c. $2932 C>T$ & p.Gln978* & Novel & Nonsense & + & \\
\hline PKD1 & 15 & c.4379_4380delTG & p.Val1460Glyfs*62 & Novel & Frameshift & + & \\
\hline PKD1 & 19 & c.7579_7580delGT & p.Val2527Leufs*67 & Novel & Frameshift & + & \\
\hline PKD1 & 22 & c.8019dupG & p.Pro2674Alafs*148 & Novel & Frameshift & + & \\
\hline PKD1 & 26 & $c .9340 C>T$ & p.Gln3114* & Novel & Nonsense & + & \\
\hline PKD1 & 42 & c.11545delG & p.Ala3849Leu*96 & Novel & Frameshift & + & \\
\hline PKD1 & 42 & C.11614G > T & p.Glu3872* & Known & Nonsense & + & PKDB [21] \\
\hline PKD1 & 43 & c.11766G > A & p.Trp3922* & Known & Nonsense & + & PKDB [1] \\
\hline PKD1 & 45 & c.12155_12156delTG & p.Val4052Glyfs*104 & Known & Frameshift & + & PKDB (unpublished) \\
\hline PKD2 & 2 & $c .667 \mathrm{G}>\mathrm{T}$ & p.Glu223* & Novel & Nonsense & + & \\
\hline PKD2 & 8 & c.1888_1891delinsGT & p.Gln630Valfs*20 & Novel & Frameshift & + & \\
\hline PKD2 & 13 & c. $2407 C>T$ & p.Arg803* & Known & Nonsense & + & PKDB [3] \\
\hline
\end{tabular}

${ }^{a}$ This mutation has been reported in one family (ATH0279) from unpublished data and is registered in PKDB by Athena Diagnostics.

the range of exons 36-46. The low mutation detection rate previously reported in Korean ADPKD patients could be explained by the fact that different mutational analyses techniques were used. In studies on other ethnic populations with different molecular diagnostic methods, mutations within PKD1 or PKD2 were detected in $63.0-89.9 \%$ of patients from Western populations [1,3,12-14,27], and 52.3-83.9\% of patients from Asian populations outside of Korea [15-17] (Table 4).

In previous studies on Korean ADPKD patients, methods other than LR-PCR and direct sequencing were used to detect mutations in PKD1 and PKD2, such as PCR and SSCP analysis for PKD1 exons 36-46 excluding the duplicated region. This approach had a mutation detection rate ranging from $6.6 \%$ (6/91 unrelated individuals) [18] to $13.7 \%$ (7/51 families) [20]. Other methods previously used include two-dimensional gene scanning followed by DGGE and direct sequencing of PKD2; a mutation detection rate of $13.0 \%$ (6/46 patients) was reported using this approach [28]. Linkage analysis using
PCR and polyacrylamide gel electrophoresis for PKD1 and $P K D 2$ resulted in a mutation detection rate of $81.3 \%$ (39/48 families) [19]. However, linkage analysis is not suitable for small families or families with a single affected individual, and becomes complicated if a de novo mutation has recently occurred in the family.

In this study, each patient had a unique pathogenic mutation; no mutations were repeatedly found in more than one individual, suggesting that a recurrent or founder mutation is not likely in this study group. This is consistent with the findings that no single mutation accounted for $>2 \%$ of all unrelated ADPKD patients in previous studies $[3,16]$. These findings support the suggestion that Korean ADPKD patients who seek a genetic diagnosis need to undergo complete mutation analyses of PKD1 and PKD2 unless causative mutations have been identified in their family members.

MLPA should be reserved for those patients in whom a mutation has not been identified by LR-PCR and direct sequencing. Large genomic rearrangements have rarely

Table 2 Likely pathogenic mutations found in this study and prediction of their pathogenicity

\begin{tabular}{|c|c|c|c|c|c|c|c|c|c|}
\hline Gene & $\begin{array}{l}\text { Exon/ } \\
\text { Intron }\end{array}$ & $\begin{array}{l}\text { cDNA } \\
\text { change }\end{array}$ & AA change & $\begin{array}{l}\text { Known/ } \\
\text { Novel }\end{array}$ & Type & $\begin{array}{l}\text { SIFT } \\
\text { VS }\end{array}$ & $\begin{array}{l}\text { PolyPhen-2 } \\
\text { VS }\end{array}$ & $\begin{array}{l}\text { GVGD } \\
\text { VS }\end{array}$ & $\begin{array}{l}\text { Family } \\
\text { history }\end{array}$ \\
\hline PKD1 & 26 & $c .9380 G>C^{a}$ & p.Gly3127Ala & Novel & Missense & 0.00 & 0.994 & C55 & + \\
\hline PKD1 & 39 & c. $11248 C>G^{a}$ & p.Arg3750Gly & Known ${ }^{b}$ & Missense & 0.00 & 0.997 & C65 & - \\
\hline PKD1 & 42 & c. $11541 C>\mathrm{G}^{\mathrm{a}}$ & p.Ser3847Arg & Novel & Missense & 0.01 & 0.805 & C65 & + \\
\hline PKD1 & IVS5 & c. $1202-9 G>A$ & p.Ala401fs & Known ${ }^{c}$ & Splice & & & & - \\
\hline
\end{tabular}

Abbreviations: AA amino acid, VS variant score, NA not applicable.

${ }^{a}$ The three novel missense variations found in this study were predicted to affect protein function by all three in-silico analyses.

${ }^{b}$ This mutation has been reported in two affected patients among four tested family members in one Czech family [27].

'This mutation has been reported in one family (ATH0012) from unpublished data by Athena Diagnostics and is registered in PKDB and classified as likely pathogenic' with the amino acid change of p.Ala401fs. Kurashige et al. also reported that this mutation created a new acceptor site seven nucleotides upstream of the original acceptor site, which was confirmed by a minigene splicing assay [17]. 
Table 3 Novel variants with unknown clinical significance found in this study

\begin{tabular}{lllllll}
\hline Gene & Exon/Intron & cDNA change & AA change & Known/Novel & HSF prediction & Family history \\
\hline PKD1 & 42 & C.11604G $>C^{\text {a }}$ & p.Thr3868 $=$ & Novel & Potentially affects enhancer motif & + \\
PKD2 & IVS1 & C.595 $+15 \mathrm{C}>\mathrm{T}$ & $?$ & Novel & No effect on normal splicing & -
\end{tabular}

Neither variations were observed in healthy controls in this study; HSF, Human Splice Finder software (http://umd.be/HSF/) used to predict splicing signals. ${ }^{a}$ This variant was observed in one patient with a family history of polycystic kidney disease (mother). Unfortunately, molecular diagnostic test results for his affected family member were not available.

been reported, and account for $<2 \%$ of the total pathogenic mutations in the PKD1 gene according to the ADPKD mutation database (PKDB). In our study, we identified one patient with a heterozygous deletion of entire PKD1 gene. This large deletion was not characterized at the nucleotide level due to the sequence complexity of the PKD1 locus.

Pathogenic mutations were not found in two of $20 \mathrm{pa}-$ tients $(10.0 \%)$ in this study, which is comparable to the results reported for a Japanese cohort (16.1\%) [17] and Western countries (10.1\%-10.9\%) [1,3]. There are several possible reasons for this finding. First, it is possible that mutations occurred within deep intronic regions, as well as in promoters and other distantly located regulatory regions not covered by the current exon-based sequencing method. Second, some of the variants that were classified as other than pathogenic mutations may in fact be hypomorphic variants with incomplete penetrance. Such variants may cause mild cystic disease, but two such variants in trans could cause typical to severe disease [7]. Otherwise, further genetic heterogeneity such as mosaicism or mutations in other genes such as HNF1 $\beta$, PRKCSH, SEC63, or PKHD1 may contribute to the cystic phenotype [7]. One of the two patients in whom no pathogenic mutations in $P K D 1$ or $P K D 2$ were found, except for the synonymous variant of c.11604G $>C$ (p.Thr3868=) in $P K D 1$, had a history of multiple liver cysts that developed in early childhood, but relatively small renal cysts in adulthood, which suggests that genes other than PKD1 and PKD2 could be involved in pathogenicity in this individual. However, this patient had a positive family history (mother with PKD), indicating that this variant could be a novel pathogenic mutation that could potentially affect splicing. Unfortunately, mutational analysis of the patient's affected mother was not performed. The other patient without identified pathogenic mutations aside from one intronic VUS (c.595 + 15C > T) in PKD2 had no family history.

As potential therapies and clinical trials with different mechanism-based drug treatments for ADPKD are being developed, more efficient, accurate, and low-cost genetic testing is needed and expected in the next decade with the advance of DNA sequencing techniques [29]. Considering the mutation spectrum in Korean patients with ADPKD, LR-PCR followed by nested PCR and direct sequence analysis of the duplicated region should be performed for an accurate molecular diagnosis and to determine the appropriate course of treatment for the disease.

Table 4 Different mutation detection rates among different study groups with different diagnostic methods

\begin{tabular}{|c|c|c|c|c|}
\hline Ethnicity & No. of patients ${ }^{a}$ & Mutation detection rate & Method & Reference \\
\hline \multicolumn{5}{|l|}{ Caucasian } \\
\hline American & 202 & $89.1 \%$ & DHPLC, LR-PCR, DS & [1] \\
\hline American & 183 & $63.0 \%$ & LR-PCR, NGS, targeted re-sequencing & {$[12]$} \\
\hline German & 93 & $64.5 \%$ & LR-PCR, DS & [13] \\
\hline German & 277 & $64.6 \%$ & DHPLC, LR-PCR, DS & [14] \\
\hline Czech & 56 & $71.0 \%$ & Linkage analysis, LR-PCR, nested PCR with HRM, DS & [27] \\
\hline French & 700 & $89.9 \%$ & LR-PCR, DS, QFM PCR or Array-CGH & [3] \\
\hline \multicolumn{5}{|l|}{ Asian } \\
\hline Chinese Hans & 65 & $52.3 \%$ & DHPLC, LR-PCR, DS & {$[15]$} \\
\hline Taiwanese & 46 & $65.0 \%$ & LR-PCR, DS, and Real time Q-PCR or MLPA & {$[16]$} \\
\hline Japanese & 161 & $83.9 \%$ & LR-PCR, DS, and Q-PCR or MLPA & [17] \\
\hline Korean & 20 & $90.0 \%$ & LR-PCR, DS, and MLPA & This study \\
\hline
\end{tabular}

Abbreviations: DHPLC, denaturing high performance liquid chromatography; LR-PCR, long range-PCR; DS, direct sequencing; NGS, next-generation DNA sequencing; HRM, high resolution melting; QFM PCR, quantitative fluorescent multiplex PCR; Array-CGH, array-comparative genomic hybridization; Q-PCR, quantitative PCR; MLPA, multiplex ligation-dependent probe amplification.

${ }^{a}$ Number of unrelated patients in the study group. 
The limitations of this study include the relatively small sample size, which limited our power to draw meaningful statistical conclusions regarding mutation prevalence and mutation detection rate. In addition, there was limited clinical data regarding genotype-phenotype correlations, and lack of experimental functional validation of novel variations. Future studies are required to investigate the significance of the VUS identified in the study.

\section{Conclusions}

PKD1 and PKD2 mutations were detected in 90.0\% of Korean ADPKD patients; mutations in PKD1 and PKD2 accounted for $83.3 \%$ and $16.7 \%$ of all mutations, respectively. Of the 14 mutations found in PKD1 (except for one large gene deletion), eight (57.1\%) mutations were located outside the range of exons 36-46. Considering the mutation spectrum in Korean patients with ADPKD, LR-PCR followed by direct sequence analysis of the duplicated region should be performed for accurate molecular diagnosis of this disease.

\section{Abbreviations}

ADPKD: Autosomal dominant polycystic kidney disease; Align-GVGD: Align Grantham Variation and Grantham Deviation, http://agvgd.iarc.fr/; CGH: Comparative genomic hybridization; DGGE: Denaturing gradient gel electrophoresis; DHPLC: Denaturing high performance liquid chromatography; ESP: Exome Sequencing Project; ESRD: End-stage renal disease; HSF: Human Splice Finder software, http://umd.be/HSF/; LR-PCR: Long range polymerase chain reaction; MLPA: Multiplex ligationdependent probe amplification; NHLBI: National Heart, Lung, and Blood Institute; PC: Polycystin; PCR: Polymerase chain reaction; PKD: Polycystic kidney disease; PKDB: ADPKD Mutation Database, http://pkdb.mayo.edu; PolyPhen-2: Polymorphism Phenotyping v.2, http://genetics.bwh.harvard.edu/ pph2/; SIFT: Sorting intolerant from tolerant, http://sift.jcvi.org/; SNP: Single nucleotide polymorphism; SSCP: Single-strand conformation polymorphism; VUS: Variant of unknown significance.

\section{Competing interests}

The authors declare that they have no competing interests.

\begin{abstract}
Authors' contributions
RC and HCP participated in the conception and design of the study and performed molecular genetic analysis of the PKD genes, collected clinical data, and prepared the manuscript. M-GL and KL helped perform molecular genetic studies of PKD genes. C-SK participated in the conception and design of the study. HCP, Y-HH and C-SK participated in recruitment of patients and collection of clinical data. C-SK, CA and Y-HH contributed to manuscript preparation and secured grant funding. C-SK and $\mathrm{Y}-\mathrm{HH}$ had full access to all data in the study and take responsibility for the integrity of the data and accuracy of the data analysis. All authors read and approved the final manuscript.
\end{abstract}

\section{Acknowledgements}

This study was supported by a grant from the Korean Health Technology R\&D Project, Ministry of Health \& Welfare, Republic of Korea (HI12C0014) and by the SMC-KIST Translational Research Program in 2013.

\section{Author details}

'Department of Laboratory Medicine and Genetics, Samsung Medical Center, Sungkyunkwan University School of Medicine, (135-710) 81 Irwon-Ro Gangnam-gu, Seoul, South Korea. ${ }^{2}$ Department of Internal Medicine, Seoul National University College of Medicine, (110-744) 28 Yeongeon-dong, Jongno-gu, Seoul, South Korea. ${ }^{3}$ Department of Internal Medicine, Eulji General Hospital, (139-872), 1306 Dunsan 2(i)-dong, Seo-gu, Daejeon, Seoul, South Korea.
Received: 10 July 2014 Accepted: 20 November 2014

Published online: 10 December 2014

\section{References}

1. Rossetti S, Consugar MB, Chapman AB, Torres VE, Guay-Woodford LM, Grantham JJ, Bennett WM, Meyers CM, Walker DL, Bae K, Zhang QJ, Thompson PA, Miller JP, Harris PC, CRISP Consortium: Comprehensive molecular diagnostics in autosomal dominant polycystic kidney disease. J Am SoC Nephrol 2007, 18(7):2143-2160.

2. Chapin HC, Caplan MJ: The cell biology of polycystic kidney disease. J Cell Biol 2010, 191(4):701-710.

3. Audrezet MP, Cornec-Le Gall E, Chen JM, Redon S, Quere I, Creff J, Benech C, Maestri S, Le Meur Y, Ferec C: Autosomal dominant polycystic kidney disease: comprehensive mutation analysis of PKD1 and PKD2 in 700 unrelated patients. Hum Mutat 2012, 33(8):1239-1250.

4. Hateboer N, v Dijk MA, Bogdanova N, Coto E, Saggar-Malik AK, San Millan $J$, Torra R, Breuning M, Ravine D: Comparison of phenotypes of polycystic kidney disease types 1 and 2. European PKD1-PKD2 Study Group. Lancet 1999, 353(9147):103-107.

5. Ravine D, Gibson RN, Walker RG, Sheffield $\amalg$, Kincaid-Smith P, Danks DM: Evaluation of ultrasonographic diagnostic criteria for autosomal dominant polycystic kidney disease 1. Lancet 1994, 343(8901):824-827.

6. Pei $Y$, Obaji J, Dupuis A, Paterson AD, Magistroni R, Dicks E, Parfrey $P$, Cramer B, Coto E, Torra R, Breuning M, Peters D, Ravine D: Unified criteria for ultrasonographic diagnosis of ADPKD. J Am Soc Nephrol 2009, 20(1):205-212.

7. Harris PC, Rossetti S: Molecular diagnostics for autosomal dominant polycystic kidney disease. Nat Rev Nephrol 2010, 6(4):197-206.

8. Cornec-Le Gall E, Audrezet MP, Chen JM, Hourmant M, Morin MP, Perrichot R, Charasse C, Whebe B, Renaudineau E, Jousset P, Guillodo MP, Grall-Jezequel A, Saliou P, Férec C, Le Meur Y: Type of PKD1 mutation influences renal outcome in ADPKD. J Am Soc Nephrol 2013, 24(6):1006-1013.

9. Symmons O, Varadi A, Aranyi T: How segmental duplications shape our genome: recent evolution of ABCC6 and PKD1 Mendelian disease genes. Mol Biol Evol 2008, 25(12):2601-2613.

10. Rossetti S, Strmecki L, Gamble V, Burton S, Sneddon V, Peral B, Roy S, Bakkaloglu A, Komel R, Winearls CG, Harris PC: Mutation analysis of the entire PKD1 gene: genetic and diagnostic implications. Am J Hum Genet 2001, 68(1):46-63.

11. Schouten JP, McElgunn CJ, Waaijer R, Zwijnenburg D, Diepvens F, Pals G: Relative quantification of 40 nucleic acid sequences by multiplex ligation-dependent probe amplification. Nucleic Acids Res 2002, 30(12):e57.

12. Rossetti S, Hopp K, Sikkink RA, Sundsbak JL, Lee YK, Kubly V, Eckloff BW, Ward CJ, Winearls CG, Torres VE, Harris PC: Identification of gene mutations in autosomal dominant polycystic kidney disease through targeted resequencing. J Am Soc Nephrol 2012, 23(5):915-933.

13. Hoefele J, Mayer K, Scholz M, Klein HG: Novel PKD1 and PKD2 mutations in autosomal dominant polycystic kidney disease (ADPKD). Nephrol Dial Transplant 2011, 26(7):2181-2188.

14. Neumann HP, Jilg C, Bacher J, Nabulsi Z, Malinoc A, Hummel B, Hoffmann MM, Ortiz-Bruechle N, Glasker S, Pisarski P, Neeff H, Krämer-Guth A, Cybulla M, Hornberger M, Wilpert J, Funk L, Baumert J, Paatz D, Baumann D, Lahl M, Felten $H$, Hausberg M, Zerres K, Eng C, Else-Kroener-Fresenius-ADPKDRegistry: Epidemiology of autosomal-dominant polycystic kidney disease: an in-depth clinical study for south-western Germany. Nephrol Dial Transplant 2013, 28(6):1472-1487.

15. Yu C, Yang Y, Zou L, Hu Z, Li J, Liu Y, Ma Y, Ma M, Su D, Zhang S: Identification of novel mutations in Chinese Hans with autosomal dominant polycystic kidney disease. BMC Med Genet 2011, 12:164.

16. Chang MY, Chen HM, Jenq CC, Lee SY, Chen YM, Tian YC, Chen YC, Hung CC, Fang JT, Yang CW, Wu-Chou YH: Novel PKD1 and PKD2 mutations in Taiwanese patients with autosomal dominant polycystic kidney disease. J Hum Genet 2013, 58(11):720-727.

17. Kurashige M, Hanaoka K, Imamura M, Udagawa T, Kawaguchi Y, Hasegawa T, Hosoya T, Yokoo T, Maeda S: A comprehensive search for mutations in the PKD1 and PKD2 in Japanese subjects with autosomal dominant polycystic kidney disease. Clin Genet 2014, in press.

18. Kim UK, Jin DK, Ahn C, Shin JH, Lee KB, Kim SH, Chae JJ, Hwang DY, Lee JG, Namkoong Y, Lee CC: Novel mutations of the PKD1 gene in Korean patients with autosomal dominant polycystic kidney disease. Mutat Res 2000, 432(1-2):39-45. 
19. Lee JG, Lee KB, Kim UK, Ahn C, Hwang DY, Hwang YH, Eo HS, Lee EJ, Kim YS, Han JS, Kim S, Lee JS: Genetic heterogeneity in Korean families with autosomal-dominant polycystic kidney disease (ADPKD): the first Asian report. Clin Genet 2001, 60(2):138-144.

20. Eo HS, Lee JG, Ahn C, Cho JT, Hwang DY, Hwang YH, Lee EJ, Kim YS, Han JS, Kim S, Lee JS, Jeoung DI, Lee SE, Kim UK: Three novel mutations of the PKD1 gene in Korean patients with autosomal dominant polycystic kidney disease. Clin Genet 2002, 62(2):169-174.

21. Tan YC, Blumenfeld JD, Anghel R, Donahue S, Belenkaya R, Balina M, Parker $T$, Levine $D$, Leonard DG, Rennert H: Novel method for genomic analysis of PKD1 and PKD2 mutations in autosomal dominant polycystic kidney disease. Hum Mutat 2009, 30(2):264-273.

22. Desmet FO, Hamroun D, Lalande M, Collod-Beroud G, Claustres M, Beroud C: Human Splicing Finder: an online bioinformatics tool to predict splicing signals. Nucleic Acids Res 2009, 37(9):e67.

23. Plon SE, Eccles DM, Easton D, Foulkes WD, Genuardi M, Greenblatt MS, Hogervorst FB, Hoogerbrugge N, Spurdle AB, Tavtigian SV: Sequence variant classification and reporting: recommendations for improving the interpretation of cancer susceptibility genetic test results. Hum Mutat 2008, 29(11):1282-1291.

24. Rossetti S, Adeva M, Kubly V, Consugar M, Torres V, Harris P: An Olmsted County population-based study indicates that PKD2 is more common than previously described. J Am Soc Nephrol 2007, 18:SA-PO93.

25. Robinson C, Hiemstra TF, Spencer D, Waller S, Daboo L, Karet Frankl FE, Sandford RN: Clinical utility of PKD2 mutation testing in a polycystic kidney disease cohort attending a specialist nephrology out-patient clinic. BMC Nephrol 2012, 13:79.

26. Barua M, Cil O, Paterson AD, Wang K, He N, Dicks E, Parfrey P, Pei Y: Family history of renal disease severity predicts the mutated gene in ADPKD. J Am Soc Nephrol 2009, 20(8):1833-1838.

27. Obeidova L, Elisakova V, Stekrova J, Reiterova J, Merta M, Tesar V, Losan F, Kohoutova M: Novel mutations of PKD genes in the Czech population with autosomal dominant polycystic kidney disease. BMC Med Genet 2014, 15:41.

28. Chung W, Kim H, Hwang YH, Kim SY, Ko AR, Ro H, Lee KB, Lee JS, Oh KH, Ahn C: PKD2 gene mutation analysis in Korean autosomal dominant polycystic kidney disease patients using two-dimensional gene scanning. Clin Genet 2006, 70(6):502-508.

29. Chang MY, Ong AC: Mechanism-based therapeutics for autosomal dominant polycystic kidney disease: recent progress and future prospects. Nephron Clin Pract 2012, 120(1):c25-c34. discussion c35.

doi:10.1186/s12881-014-0129-y

Cite this article as: Choi et al:: Identification of novel PKD1 and PKD2

mutations in Korean patients with autosomal dominant polycystic kidney disease. BMC Medical Genetics 2014 15:129.

\section{Submit your next manuscript to BioMed Central and take full advantage of:}

- Convenient online submission

- Thorough peer review

- No space constraints or color figure charges

- Immediate publication on acceptance

- Inclusion in PubMed, CAS, Scopus and Google Scholar

- Research which is freely available for redistribution 